\title{
Significance of TP53 mutation in bladder cancer disease progression and drug selection
}

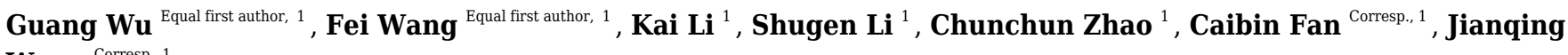 \\ Wang ${ }^{\text {Corresp. } 1}$ \\ ${ }^{1}$ The Affiliated Suzhou Hospital of Nanjing Medical University, Suzhou, China \\ Corresponding Authors: Caibin Fan, Jianqing Wang \\ Email address: fancaibin@sina.com, jqwang14@fudan.edu.cn
}

Background: The tumor protein p53 (TP53) mutant is one of the most frequent mutant gene in bladder cancer. In this study, we assessed the importance of TP53 mutation in bladder cancer progression and drug selection, and identified potential pathways and core genes associated with the underlying mechanisms.

Methods: Gene expression data used in this study were downloaded from the cancer Genome Atlas (TCGA) and cBioportal databases. Drug sensitivity data were obtained from GDSC (The Genomics of Drug Sensitivity in Cancer). We did functional enrichment analysis by Gene set enrichment analysis (GSEA) and the database for annotation, visualization and integrated discovery (DAVID).

Results: We found TP53 mutation in 50\% bladder cancer patients. Patients with TP53 mutation were associated with lower TP53 mRNA expression level, more advanced tumor stage and higher histologic grade. Three drugs, mitomycin-C, doxorubicin and gemcitabine, were especially more sensitive to bladder cancer with TP53 mutation. As for the mechanisms, we identified 863 differentially expressed genes. Functional enrichment analysis suggested that DEGs were primarily enriched in multiple metabolic progressions, chemical carcinogenesis and cancer related pathways. The protein-protein interaction network identified the top ten hub genes. Our results have suggested the significance of TP53 mutation in disease progression and drug selection in bladder cancer, and identified multiple genes and pathways related in such program, offering novel basis for bladder cancer individualized treatment. 
1 Significance of TP53 mutation in bladder cancer disease progression and drug selection

2 Running title: TP53 mutation in bladder cancer

3 Guang $\mathrm{Wu}{ }^{1 \S}$, Fei Wang ${ }^{1 \S}, \mathrm{Kai} \mathrm{Li}^{1 \S}$, Shugen $\mathrm{Li}^{1}$, Chunchun $\mathrm{Zhao}^{1}$, Caibin Fan ${ }^{1 *}$ and Jianqing

4 Wang $^{1 *}$

$5{ }^{1}$ Department of Urology, The Affiliated Suzhou Hospital of Nanjing Medical University

6

$7 \S$ First contributors for this work.

$8 *$ *Correspondence to:

9 Jianqing Wang, Department of Urology, The Affiliated Suzhou Hospital of Nanjing Medical

10 University, 26 Daoqian Rd, Suzhou, Jiangsu 215000, PR China; Tel: +86-512-62362011; E-mail:

11 jqwang14@fudan.edu.cn

12 Caibin Fan: fancaibin@sina.com

13 


\section{Abstract}

15 Background: The tumor protein p53 (TP53) mutant is one of the most frequent mutant gene in bladder cancer. In this study, we assessed the importance of TP53 mutation in bladder cancer progression and drug selection, and identified potential pathways and core genes associated with the underlying mechanisms.

Methods: Gene expression data used in this study were downloaded from the cancer Genome Atlas (TCGA) and cBioportal databases. Drug sensitivity data were obtained from GDSC (The Genomics of Drug Sensitivity in Cancer). We did functional enrichment analysis by Gene set enrichment analysis (GSEA) and the database for annotation, visualization and integrated discovery (DAVID).

Results: We found TP53 mutation in 50\% bladder cancer patients. Patients with TP53 mutation were associated with lower TP53 mRNA expression level, more advanced tumor stage and higher histologic grade. Three drugs, mitomycin-C, doxorubicin and gemcitabine, were especially more sensitive to bladder cancer with TP53 mutation. As for the mechanisms, we identified 863 differentially expressed genes. Functional enrichment analysis suggested that DEGs were primarily enriched in multiple metabolic progressions, chemical carcinogenesis and cancer related pathways. The protein-protein interaction network identified the top ten hub genes.

Our results have suggested the significance of TP53 mutation in disease progression and drug selection in bladder cancer, and identified multiple genes and pathways related in such program, offering novel basis for bladder cancer individualized treatment.

Keywords: bladder cancer, TP53 mutation, TCGA, RNA sequencing, bioinformatics analysis, 
35 drug selection

36

37 
Introduction

39 Carcinoma of the urinary bladder (bladder cancer) is one of the worldwide most common

40 malignancies, causing almost 150,000 deaths every year (Siegel et al. 2016). Bladder cancer is

41 prone to recurrence and has strong invasiveness, which means that about $25 \%$ of patients

42 develop into muscle-invasive (MIBC) or metastatic disease as initial diagnosis or during the

43 treatment and have a worse prognosis. Transurethral resection of the bladder (TURB) is the

44 primary treatment of initial non muscle-invasive bladder cancer (NMIBC). After TURB,

45 intravesical chemotherapy is the critical adjuvant therapy in all patients to prevent recurrence.

46 Until now, surgery and chemotherapy are the primary treatments of MIBC. Today,

47 individualized treatment, including molecular pathological diagnosis and individualized

48 medicine testing, can help clinicians select target chemotherapy drugs according to the

49 characteristics of patient's tumor cells. Such individualized treatment could achieve better

50 therapeutic effect, delay disease progression, and improve the prognosis. Therefore, to identify

51 the feature of bladder cancer patients could provide candidate targets and strategies for

52 individualized treatment.

53 Tumor protein p53 (TP53) is now identified as a tumor suppressor in various tumor types. It can

54 regulate target gene expression in order to play multiple roles in coping with complicated cellular

55 progression, resulting in transcriptional activation, senescence, apoptosis or changes in

56 metabolism and cell cycle (Vousden \& Prives 2009). Mutation in TP53 is frequently found in

57 human cancer, including bladder cancer. Previous studies have shown that nearly half of the

MIBC samples had TP53 mutation, and that TP53 function was inactivated in 76\% of samples 
59 (Network 2014; Rentsch et al. 2017). TP53 mutation and TP53 related pathways could serve as 60 driver mutations in bladder cancer, which promotes disease progression and influences cancer 61 prognosis and therapeutic strategy (Cazier et al. 2014; Lang et al. 2004). Mutant TP53 also 62 induces the activation of mechanisms in cancer initiation and progression, which contributes to 63 poor disease outcome (Kim et al. 2015; Smith et al. 2002). Many researchers have shown that 64 mutant TP53 could accelerate metastatic tumor cell proliferation and promote the metastatic 65 ability (Morton et al. 2010). Moreover, TP53 status has been shown to influence chemotherapy 66 and drug sensitivity in bladder cancer (Pandey et al. 2018; Ye et al. 1998). As TP53 mutation is 67 the most frequently detected mutation in bladder cancer patients (Lamy et al. 2006), we intend to study whether clustering analysis of data from molecular profiling would define TP53 mutant bladder tumors as a subgroup to help identify some novel individualized treatment methods.

In the present study, we explored GDSC database to estimate the significance of TP53 mutation in bladder cancer drug selection, and analyzed RNA sequencing (RNA-Seq) dataset of MIBC to find out the role of TP53 mutation in disease progression, providing novel individualized treatment options. Meanwhile, the identification of critical pathways and core genes associated with TP53 mutation could uncover the potential mechanisms and therapeutic targets. 


\section{Materials and methods}

\section{Analysis of the GDSC database}

GDSC (The Genomics of Drug Sensitivity in Cancer) project is setup for cancer molecular therapeutics and mutation exploration (Vanden Heuvel et al. 2018). Public online platform has been developed to help researchers to analyze the astronomical data matrix and download data (Yang et al. 2013). In this article, we first searched compounds with significant selectivity for TP53 mutation, and then tried to narrow the hits by check the sensitivity only in bladder cancer cells. The volcano plots, scatter plots, and the statistical analysis were downloaded directly from the GDSC online platform.

\section{Gene set enrichment analysis (GSEA)}

We analyzed GSEA to find out the differences in gene mRNA expression levels of biological annotation and pathways to help understand the effect of TP53 mutation on biological function gene sets in bladder cancer patients.

\section{Identification of differentially expressed genes (DEGs)}

We used EdgeR to examine differential gene expression between TP53 mutation and wild-type bladder cancer patients (McCarthy et al. 2012; Robinson et al. 2010). DEGs were identified with the following criterion: $\mid$ fold change $(\mathrm{FC}) \mid \geq 2$; P-value $<0.05$. 
97 Here, we used the database for annotation, visualization and integrated discovery (DAVID) to

98

99

100

101

102

103

104

105

106

107

108

109

110 complete gene functional analysis (Dennis et al. 2003). GO (Gene Ontology) and KEGG (Kyoto

Encyclopedia of Genes and Genomes) analysis were uploaded to DAVID website for enrichment analysis, while specifying a P-value $<0.05$ for statistical significance. DAVID: https:/ /david.ncifcrf.gov/.

\section{Statistical analysis}

We used the Student's t-test to compare the mRNA expression level in different TP53 status bladder cancer tissue. The association between TP53 mutation and the clinic parameters were evaluated by a $\chi^{2}$ test. The Kaplan-Meier method with log-rank test was used for calculating the clinical prognosis between by Graphpad. In GSEA, enrichment results satisfying a nominal Pvalue cutoff of 0.05 with a false discovery rate (FDR q-val) $<0.25$ were considered statistically significant. All the statistical analyses were conducted with R 3.3. 0 and Graphpad. A value of P $<0.05$ was considered statistically significant. 
111

112

113

114

115

116

117

118

119

120

121

122

123

124

125

126

127

128

129

130

131

\section{Results}

\section{TP53 mutation is more common in MIBC}

We first downloaded the information and complete follow-up profiles of 408 MIBC patients and their cancer tissue expression data from TCGA database. There were 206 patients $(49.5 \%)$ with TP53 mutation (Figure 1 A). Main mutation types were amplification, truncating, deep deletion, inframe mutation and missense mutations spanning over entire gene (Figure $1 \mathrm{~B}$ ). To initially explore whether TP53 mutation is an important factor in the progression of bladder cancer, we also analyzed the proportion of TP53 mutation in NMIBC. However, there were only $21 \%$ patients with less TP53 mutation types in another dataset of NMIBC, indicating the possibility that TP53 mutation might contribute to the progression to muscle-invasive disease, and that TP53 mutation might be a potential mark of bladder cancer being easy invasiveness (Figure $1 \mathrm{C}$, D).

\section{TP53 mutation in bladder cancer progress and prognosis}

We next explored the influence of TP53 mutation on bladder cancer development and prognosis.

We first compared the clinical information of bladder cancer patients in the two groups. After analyzing, we found that patients with TP53 mutation have higher neoplasm histologic grade and more advanced tumor stage, indicating that TP53 mutation might contribute to the disease progression (Table 1).

We then investigated TP53 mRNA expression in TP53 wild type and mutated groups. Results indicated that TP53 expression level was lower in mutated bladder cancer patients' tissue (Figure 
1322 A). However, TP53 mutation had no effect on disease prognosis of survive (Figure 2 B) and

133 recurrence (Figure $2 \mathrm{C}$ ). Results above indicated that TP53 mutation may contribute to bladder

134 cancer disease progression, but not the prognosis. Early intervention may be benefit to such 135 patients.

136

137

Bladder cancer cells with TP53 mutation are sensitive to mitomycin-C, doxorubicin and

gemcitabine

139 Chemotherapy is one of the chief treatments for bladder cancer. Therefore, the sensitivity to chemotherapy drugs determines the therapeutic effect. In addition to TP53 mutation on cancer progression, we also investigated whether TP53 mutation induces drug resistance in bladder cancer. In order to explore the role of TP53 mutation in drug sensitivity and find out specific inhibitors for individualized treatment, we explored the GDSC database to investigate whether TP53 mutated patients have selective compounds in bladder cancer. Results indicated that bladder cancer cells that harbor TP53 mutation, not other types of tumors, were significant sensitive to mitomycin-c, doxorubicin and gemcitabine (Figure 3), making them potential target compounds. As a result, mitomycin-C, doxorubicin and gemcitabine conferred potential individualized compounds for bladder cancer patients with TP53 mutation.

\section{Results of gene set enrichment analysis}

Our results above showed that TP53 mutation plays an important role in bladder cancer disease development and drug selection. Then we analyzed the effects of TP53 mutation on cellular 
153 process to investigate the underlying mechanism. We first used GSEA approach to analyze

154 biological functional gene sets. GSEA (Gene probe enrichment analysis) is a method to reveal

155 the metabolic pathway enrichment situation of all the gene expression of the selected sample for

156 enrichment analysis. Enrichment results satisfying a nominal P-value cutoff of 0.05 with a false

157 discovery rate (FDR q-val) $<0.25$ were considered statistically significant in the present study.

158 We found 23 gene sets that were significantly enriched (Table s1) and showed the 16 most

159

160

161

162

163

164

165

166

167

168

169

170

171

172

173

enriched gene sets obtained by normalized enrichment score (NES) value ranking in Figure 4.

Among the 16 gene sets, DNA repair, G2M checkpoint, glycolysis, PI3K-AKT-mTOR signaling,

MYC targets, mTORC1 signaling, cholesterol homeostasis, protein secretion, peroxisome, E2F

targets, androgen response, reactive oxygen species (ROS) pathway and mitotic spindle are closely related to tumorigenesis. These results suggest that TP53 mutation may promote bladder cancer progression by influencing pathways in cancer, ROS, metabolism and DNA repair.

\section{Identification of DEGs}

We next identified the DEGs to find out the pathways and genes implicated in TP53 mutation.

Based on the in silico analysis, 863 genes were identified as DEGs, among which 678 were upregulated and 185 were downregulated. The volcano plot of the DEGs is shown in Figure 5 A.

1 GO and KEGG analysis of DEGs

72 Then we uploaded the 863 DEGs online for further functional enrichment analyses using DAVID. Results of GO analysis (Figure $5 \mathrm{~B}$ ) suggested significant enrichment in positive 
174 regulation of immune response, calcium ion-regulated exocytosis of neurotransmitter, regulation 175 of ion transmembrane transport, regulation of membrane potential, adenylate cyclase-activating 176 adrenergic receptor signaling pathway, xenobiotic metabolic process, acute-phase response, 177 detection of chemical stimulus involved in sensory perception of smell, angiotensin maturation, 178 adult walking behavior, layer formation in cerebral cortex, regulation of calcium ion-dependent 179 exocytosis and G-protein coupled receptor signaling pathway.

180 Moreover, KEGG pathway analysis showed significant enrichment in chemical carcinogenesis, 181 metabolism of xenobiotics by cytochrome $\mathrm{P} 450$, retinol metabolism, drug metabolism 182 cytochrome P450, neuroactive ligand-receptor interaction, steroid hormone biosynthesis, 183 olfactory transduction, glioma, transcriptional misregulation in cancer, cytokine-cytokine 184 receptor interaction, bladder cancer, gastric acid secretion and Jak-Stat signaling pathway 185 (Figure $5 \mathrm{C}$ ).

186 We then used the Search Tool for the Retrieval of Interacting Genes (STRING) database to 187 investigate the interaction and hub genes of DEGs. The top ten genes ranked by degree were 188 identified as hub genes, including EGFR, GNG4, PRKACG, CSF2, CTAG1A, CTAG1B, 189 BAGE5, GABBR2, F2, PVALB. EGFR had the highest degree of nodes among the hub genes 190 with 26. 
192

193

194 195 196 197 198 199 200 201 202 203 204 205 206 207 208 209 210 211 212

\section{Discussion}

TP53 acts as a lipid phosphatase, removing the phosphate in the D3 position of the inositol ring.

Previous studies showed that the major function of TP53 relies on its phosphatase activity. TP53 showed antagonism of the PI3K/AKT pathway, while AKT-independent function was also found in other studies (Maehama et al. 2001). TP53 mutation is frequently found in multiple human cancers, including bladder cancer. TP53 mutation and related pathways could be drivers in bladder cancer initiation (Cazier et al. 2014; Williamson et al. 1994). TP53 mutation and other critical gene mutations have been shown to alter multiple cancer related pathways in bladder cancer and thus promote disease progression, which makes it a potential therapeutic target (Bakkar et al. 2003; Choi et al. 2014; Gui et al. 2011; Liu \& Kwiatkowski 2015). Here in the present study, we evaluated the clinical significance of TP53 mutation in bladder cancer to provide some basis for individualized treatment. Mechanisms of TP53 mutation promoting disease progression were also analyzed.

In clinical terms, patients with MIBC have more TP53 mutations than patients with NMIBC (Figure 1). Clinical characteristic analysis of bladder cancer patients in both groups showed higher neoplasm histologic grade and more advanced tumor stage in bladder cancer patients with TP53 mutation (Table 1). Results above suggest the role of TP53 mutation as an indicator to advanced tumor with malignant potential, and that TP53 mutation may be involved in the progression of bladder cancer. In addition, detection of tumor gene mutations now can help clinicians judge the prognosis of patients after surgery and recommend better individualized treatment strategies. Our results showed the evidence that bladder cancer patients with TP53 
213 mutation might need early and comprehensive intervention to live longer. Especially, bladder

214 cancer patients with TP53 mutation may require early systemic chemotherapy, other than merely

215 intravesical chemotherapy, to cope with disease progression. Besides, data from GDSC show

216 preliminary evidence that mitomycin-c, doxorubicin and gemcitabine exhibit sensitivity for

217 bladder cancer cells that harbor TP53 mutation, which provides evidence for the use of specific

218 anti-tumor drugs to such patient in clinical practice (Figure 3). Mitomycin-c, doxorubicin and

219 gemcitabine are the first line chemotherap drugs, which could decrease cell proliferation and

metastasis in human bladder cancer cell lines and have been shown to decrease the number of

subsequent recurrences and increase the recurrence-free interval in bladder cancer by multiple

mechanisms (such as hyperthermia) (Galsky et al. 2015; Humphrey \& Mann 1949; Sternberg

2000; Tolley et al. 1996; van der Heijden et al. 2004; von der Maase et al. 2000; Yu et al. 2018).

The effects of mitomycin-c, doxorubicin and gemcitabine on cells with or without mutated TP53

have been well established. Mitocmycin-c has been reported to induce TP53 independent cell

death and the expression of TP53 was not associated with the outcomes of patient treated with

mitomycin C (Seo et al. 2010). Early preliminary researches have indicated that doxorubicin has

TP53-dependent cell death response and TP53 mutantin could enhance chemosensitivity of

doxorubicin in bladder cancer cells (Bilim et al. 2000; Chang \& Lai 2001), while gemcitabine is

effective in bladder cell lines independent of TP53 status (Fechner et al. 2003). However, some

results are still controversial and various confounding factors could influence drug sensitivity .

MDM2 is an oncogene that acts by suppressing TP53 function in multiple cancer types (Toi et al.

1997). MDM2 overexpression has been found in some bladder cancer cells and contributes to 
234 doxorubicin resistance in bladder cancer, which could be a confounding factor to conclude that

235 doxorubicin would be a suitable drug to target tumors with mutant TP53 (Shiina et al. 1999;

236 Smith et al. 2003). The expression of pro-oncogenic RUNX2 could also confuse the results of

237 gemcitabine treatment in cancer with TP53 mutation (Ozaki et al. 2016; Ozaki et al. 2018).

238 Besides, mutant TP53 could produce a stable protein, so further analyses would focus on the

239 protein level to conclude more accurate results. To sum up, our results could provide some novel

240 preliminary evidence in developing better individualized treatment strategies. Until now, the

241 research data of relationship between TP53 mutation and bladder cancer chemosensitivity are

242 still limited and the final conclusion is still controversial owing to confounding factors. Because

243 of the critical role of chemotherapy in bladder cancer treatment, further basic and clinical

244 investigations are necessary to determine the role of TP53 mutation in chemotherapy and drug

245 selection.

246 To investigate the underlying mechanisms, we analyzed the gene expression data of MIBC to

247 identify the key pathways and core genes associated with TP53 mutation. In the present study,

248 we combined the results of GO/KEGG and GSEA to have the final conclusion. The input

249 variable of GSEA is the gene expression level, and the input variable of GO/ KEGG pathway

250 enrichment analysis is the gene list. Both analyses can screen out the significantly enriched

251 pathway, the difference is that GSEA is for all genes, GO/ KEGG is for differential gene

252 enrichment. Results of GSEA analysis suggest that TP53 mutation was mainly associated with

253 multiple cancer related pathways, cell proliferation and division, DNA repair, ROS and

254 metabolism (Figure 4). All programs above are all important contributors to bladder cancer 
initiation and development. Excessive accumulation of ROS could be a byproduct intracellular

256 by mitochondria and other cellular elements and exogenously by pollutants, tobacco, smoke,

257 drugs, xenobiotics, and radiation, thus promoting cancer through modulating various cell

258 signaling pathways and inducing epigenetic changes in genes in various cancer types, including

bladder cancer (Prasad et al. 2017). Metastatic bladder cancer cells show different antioxidant

expression profile, resulting in an increase in ROS production network (Hempel et al. 2009;

Miyajima et al. 1997). As TP53 is one of the inhibitor of PI3K-AKT-mTOR signaling pathway,

TP53 mutation influences the mTORC1 signaling pathway activity definitely.

We next identified the DEGs and carried out the functional enrichment analyses (Figure 5).

Results show 863 genes as DEGs with different TP53 mutation status. Enrichment analysis

suggested that DEGs in TP53 mutation bladder cancer patients were related to multiple cellular

programs. Among all programs, we found chemical carcinogenesis as the top KEGG term. As

we all know, the occurrence of bladder cancer is closely related to the long-term stimulation of

bladder cells by chemical carcinogens. Our results indicate the possibility that TP53 mutation

might aggravate the carcinogenic effects of various carcinogens on bladder cells. On the other

hand, it is very likely that it is the large number of chemical carcinogens that stimulate and

induce mutations in TP53. The results of KEGG/ GO combined with GSEA and the clinical

impact of TP53 on the disease progression highlights the importance of early prevention of

bladder cancer, and the significant role of metabolism and cancer related pathways in bladder

cancer development.

In PPI network analysis, we found out the top 10 hub genes with the highest degree of interaction. 
276 Amplification and mutations of epidermal growth factor receptor (EGFR) have been shown to be

277 driving events in several solid tumors including bladder cancer (Villares et al. 2007). Also,

278 bladder cancer cells are sensitive to treatment with drugs that targeting the EGFR pathway,

279 which has been shown to be a therapeutic target in dealing with advanced bladder cancer (Mason

280 et al. 2009).

281 Our study contained one limitation. In our study, we can only compare the mutation frequency and type of TP53 in MIBC and NMIBC. Because of the lack of clinical characteristics, prognosis and gene expression data of TP53 mutated NMIBC in TCGA, we failed to further compare these attributes. To further distinguish these different types of bladder cancer needs more data and further study.

\section{Conclusions}

In conclusion, this study found out the clinical significance of TP53 mutation in bladder cancer, and the main pathways and genes associated with TP53 mutation, which may facilitate developing early intervention and providing better therapeutic strategies against such special subtype of bladder cancer. Furthermore, the mechanism and validation of TP53 mutation in bladder cancer still need further research in clinical and molecular biology experiments.

Declaration of conflicting interests

The author(s) declared no potential conflicts of interest with respect to the research, authorship, and/or publication of this article. 


\section{Data Availability}

299

MIBC RNA-Seq dataset was downloaded directly from TCGA database (accession number:

300

TCGA-BLCA). The corresponding clinic information was obtained from the cBioPortal for

301

Cancer Genomics website (Bladder Cancer; TCGA, Cell 2017). The mutation information of

302

NMIBC was also obtained from the cBioPortal for Cancer Genomics website (Nonmuscle

303

Invasive Bladder Cancer; MSK Eur Urol 2017). DOI: 10.1158/2159-8290.CD-12-0095

304

Websites:

305

TCGA: https://portal.gdc.cancer.gov/projects/TCGA-BLCA;

306

cBioPortal: http://www.cbioportal.org/study/summary?id=blca tcga pub 2017;

307

NMIBC: $\underline{\text { http://www.cbioportal.org/study/summary?id=blca nmibc } 2017}$.

308

309

\section{Funding}

310

The authors disclosed receipt of the following financial support for the research and publication

of this article: This study was supported by National Natural Science Foundation of China (grant

no. 81802565); Natural Science Foundation of Jiangsu Province (grant no. BK20180216); Key

Project of the Scientific Research Project of Nanjing Medical University Affiliated Suzhou

Hospital (grant no. szslyy2017005).

\section{Acknowledgements}

We acknowledge the cBioPortal for Cancer Genomics site and the TCGA Research Network for 
318 generating TCGA datasets. 


\section{FIGURE LEGENDS}

320 Fig. 1 Mutation frequency and types of TP53 in bladder cancer from the cancer Genome Atlas

321 (TCGA) database.

322 A. Mutation frequency of TP53 in MIBC.

323 B. TP53 protein mutation diagram showed the mutation types of TP53 in MIBC. P53_TAD: P53

324 transactivation motif, P53 tetramer: P53 tetramerisation motif.

325

C. Mutation frequency of TP53 in NMIBC.

326

D. TP53 protein mutation diagram showed the mutation types of TP53 in NMIBC. P53_TAD:

P53 transactivation motif, P53 tetramer: P53 tetramerisation motif.

Fig. 2 TP53 mutation and bladder cancer prognosis.

A. Correlation between TP53 mutation and mRNA expression.

B. C. Kaplan-Meier survival and disease recurrence curves for bladder cancer patients stratified

by TP53 mutation.

Fig. 3 TP53 mutation influences drug selection of bladder cancer.

A. Volcano plotting showed that bladder cancer with TP53 mutation was significantly sensitive to mitomycin-c, doxorubicin and gemcitabine.

B- G. Reproduction of GDSC database showed that bladder cancer cells with TP53 mutation, but not cancer of other types, was significantly inhibited by Mitomycin-C, Doxorubicin and

Gemcitabine. NS: not significant, $* P<0.05, * * P<0.01, * * * P<0.001$. 
341 Fig. 4 GSEA results of TP53 mutation in bladder cancer patients.

342

343 Fig. 5 DAVID enrichment results of differentially expressed genes.

344 A. Volcano plot for differentially expressed genes.

345

B. GO enrichment terms of differentially expressed genes.

346

C. KEGG pathway analysis of differentially expressed genes.

347

348 


\section{REFERENCE}

Bakkar AA, Wallerand H, Radvanyi F, Lahaye JB, Pissard S, Lecerf L, Kouyoumdjian JC, Abbou CC, Pairon JC, Jaurand MC, Thiery JP, Chopin DK, and de Medina SG. 2003. FGFR3 and TP53 gene mutations define two distinct pathways in urothelial cell carcinoma of the bladder. Cancer Res 63:8108-8112.

Bilim V, Kawasaki T, Takahashi K, and Tomita Y. 2000. Adriamycin induced G2/M cell cycle arrest in transitional cell cancer cells with wt p53 and p21(WAF1/CIP1) genes. J Exp Clin Cancer Res 19:483-488.

Cazier JB, Rao SR, McLean CM, Walker AK, Wright BJ, Jaeger EE, Kartsonaki C, Marsden L, Yau C, Camps C, Kaisaki P, Taylor J, Catto JW, Tomlinson IP, Kiltie AE, and Hamdy FC. 2014. Whole-genome sequencing of bladder cancers reveals somatic CDKN1A mutations and clinicopathological associations with mutation burden. Nat Commun 5:3756. 10.1038/ncomms4756

Chang FL, and Lai MD. 2001. Various forms of mutant p53 confer sensitivity to cisplatin and doxorubicin in bladder cancer cells. J Urol 166:304-310.

Choi W, Porten S, Kim S, Willis D, Plimack ER, Hoffman-Censits J, Roth B, Cheng T, Tran M, Lee IL, Melquist J, Bondaruk J, Majewski T, Zhang S, Pretzsch S, Baggerly K, Siefker-Radtke A, Czerniak B, Dinney CP, and McConkey DJ. 2014. Identification of distinct basal and luminal subtypes of muscle-invasive bladder cancer with different sensitivities to frontline chemotherapy. Cancer Cell 25:152-165. 10.1016/j.ccr.2014.01.009

Dennis G, Jr., Sherman BT, Hosack DA, Yang J, Gao W, Lane HC, and Lempicki RA. 2003. DAVID: Database for Annotation, Visualization, and Integrated Discovery. Genome Biol 4:P3.

Fechner G, Perabo FG, Schmidt DH, Haase L, Ludwig E, Schueller H, Blatter J, Mller SC, and Albers P. 2003. Preclinical evaluation of a radiosensitizing effect of gemcitabine in p53 mutant and p53 wild type bladder cancer cells. Urology 61:468-473. 10.1016/s0090-4295(02)02156-8

Galsky MD, Pal SK, Chowdhury S, Harshman LC, Crabb SJ, Wong YN, Yu EY, Powles T, Moshier EL, Ladoire S, Hussain SA, Agarwal N, Vaishampayan UN, Recine F, Berthold D, Necchi A, Theodore C, Milowsky MI, Bellmunt J, and Rosenberg JE. 2015. Comparative effectiveness of gemcitabine plus cisplatin versus methotrexate, vinblastine, doxorubicin, plus cisplatin as neoadjuvant therapy for muscle-invasive bladder cancer. Cancer 121:2586-2593. 10.1002/cncr.29387

Gui Y, Guo G, Huang Y, Hu X, Tang A, Gao S, Wu R, Chen C, Li X, Zhou L, He M, Li Z, Sun X, Jia W, Chen J, Yang S, Zhou F, Zhao X, Wan S, Ye R, Liang C, Liu Z, Huang P, Liu C, Jiang H, Wang Y, Zheng H, Sun L, Liu X, Jiang Z, Feng D, Wu S, Zou J, Zhang Z, Yang R, Zhao J, Xu C, Yin W, Guan Z, Ye J, Zhang H, Li J, Kristiansen K, Nickerson ML, Theodorescu D, Li Y, Zhang X, Li S, Wang J, Yang H, and Cai Z. 2011. Frequent mutations of chromatin remodeling genes in transitional cell carcinoma of the bladder. Nat Genet 43:875-878. 10.1038/ng.907

Hempel N, Ye H, Abessi B, Mian B, and Melendez JA. 2009. Altered redox status accompanies progression to metastatic human bladder cancer. Free Radic Biol Med 46:42-50. 10.1016/j.freeradbiomed.2008.09.020

Humphrey GF, and Mann T. 1949. Studies on the metabolism of semen; citric acid in semen. Biochem J 44:97-105.

Kim MP, Zhang Y, and Lozano G. 2015. Mutant p53: Multiple Mechanisms Define Biologic Activity in Cancer. Front Oncol 5:249. 10.3389/fonc.2015.00249

Lamy A, Gobet F, Laurent M, Blanchard F, Varin C, Moulin C, Andreou A, Frebourg T, and Pfister C. 2006. Molecular profiling of bladder tumors based on the detection of FGFR3 and TP53 mutations. J Urol 176:2686-2689. 
10.1016/j.juro.2006.07.132

Lang GA, Iwakuma T, Suh YA, Liu G, Rao VA, Parant JM, Valentin-Vega YA, Terzian T, Caldwell LC, Strong LC, ElNaggar AK, and Lozano G. 2004. Gain of function of a p53 hot spot mutation in a mouse model of LiFraumeni syndrome. Cell 119:861-872. 10.1016/j.cell.2004.11.006

Liu Y, and Kwiatkowski DJ. 2015. Combined CDKN1A/TP53 mutation in bladder cancer is a therapeutic target. Mol Cancer Ther 14:174-182. 10.1158/1535-7163.MCT-14-0622-T

Maehama T, Taylor GS, and Dixon JE. 2001. PTEN and myotubularin: novel phosphoinositide phosphatases. Annu Rev Biochem 70:247-279. 10.1146/annurev.biochem.70.1.247

Mason RA, Morlock EV, Karagas MR, Kelsey KT, Marsit CJ, Schned AR, and Andrew AS. 2009. EGFR pathway polymorphisms and bladder cancer susceptibility and prognosis. Carcinogenesis 30:1155-1160. 10.1093/carcin/bgp077

McCarthy DJ, Chen Y, and Smyth GK. 2012. Differential expression analysis of multifactor RNA-Seq experiments with respect to biological variation. Nucleic Acids Res 40:4288-4297. 10.1093/nar/gks042

Miyajima A, Nakashima J, Yoshioka K, Tachibana M, Tazaki H, and Murai M. 1997. Role of reactive oxygen species in cis-dichlorodiammineplatinum-induced cytotoxicity on bladder cancer cells. Br J Cancer 76:206-210. 10.1038/bjc.1997.363

Morton JP, Timpson P, Karim SA, Ridgway RA, Athineos D, Doyle B, Jamieson NB, Oien KA, Lowy AM, Brunton VG, Frame MC, Evans TR, and Sansom OJ. 2010. Mutant p53 drives metastasis and overcomes growth arrest/senescence in pancreatic cancer. Proc Natl Acad Sci U S A 107:246-251. 10.1073/pnas.0908428107

Network CGAR. 2014. Comprehensive molecular characterization of urothelial bladder carcinoma. Nature 507:315322. 10.1038/nature12965

Ozaki T, Nakamura M, Ogata T, Sang M, Yoda H, Hiraoka K, and Shimozato O. 2016. Depletion of pro-oncogenic RUNX2 enhances gemcitabine (GEM) sensitivity of p53-mutated pancreatic cancer Panc-1 cells through the induction of pro-apoptotic TAp63. Oncotarget 7:71937-71950. 10.18632/oncotarget.12433

Ozaki T, Yu M, Yin D, Sun D, Zhu Y, Bu Y, and Sang M. 2018. Impact of RUNX2 on drug-resistant human pancreatic cancer cells with p53 mutations. BMC Cancer 18:309. 10.1186/s12885-018-4217-9

Pandey S, Bourn J, and Cekanova M. 2018. Mutations of p53 decrease sensitivity to the anthracycline treatments in bladder cancer cells. Oncotarget 9:28514-28531. 10.18632/oncotarget.25530

Prasad S, Gupta SC, and Tyagi AK. 2017. Reactive oxygen species (ROS) and cancer: Role of antioxidative nutraceuticals. Cancer Lett 387:95-105. 10.1016/j.canlet.2016.03.042

Rentsch CA, Muller DC, Ruiz C, and Bubendorf L. 2017. Comprehensive Molecular Characterization of Urothelial Bladder Carcinoma: A Step Closer to Clinical Translation? Eur Urol 72:960-961. 10.1016/j.eururo.2017.06.022

Robinson MD, McCarthy DJ, and Smyth GK. 2010. edgeR: a Bioconductor package for differential expression analysis of digital gene expression data. Bioinformatics 26:139-140. 10.1093/bioinformatics/btp616

Seo HK, Cho KS, Chung J, Joung JY, Park WS, Chung MK, and Lee KH. 2010. Prognostic value of p53 and Ki-67 expression in intermediate-risk patients with nonmuscle-invasive bladder cancer receiving adjuvant intravesical mitomycin C therapy. Urology 76:512 e511-517. 10.1016/j.urology.2010.04.040

Shiina H, Igawa M, Shigeno K, Yamasaki Y, Urakami S, Yoneda T, Wada Y, Honda S, and Nagasaki M. 1999. Clinical significance of $\mathrm{mdm} 2$ and $\mathrm{p} 53$ expression in bladder cancer. A comparison with cell proliferation and apoptosis. Oncology 56:239-247. 10.1159/000011971 
Siegel RL, Miller KD, and Jemal A. 2016. Cancer statistics, 2016. CA Cancer J Clin 66:7-30. 10.3322/caac.21332

Smith G, Carey FA, Beattie J, Wilkie MJ, Lightfoot TJ, Coxhead J, Garner RC, Steele RJ, and Wolf CR. 2002. Mutations in APC, Kirsten-ras, and p53--alternative genetic pathways to colorectal cancer. Proc Natl Acad Sci U S A 99:9433-9438. 10.1073/pnas.122612899

Smith ND, Rubenstein JN, Eggener SE, and Kozlowski JM. 2003. The p53 tumor suppressor gene and nuclear protein: basic science review and relevance in the management of bladder cancer. J Urol 169:1219-1228. 10.1097/01.ju.0000056085.58221.80

Sternberg CN. 2000. Gemcitabine in bladder cancer. Semin Oncol 27:31-39.

Toi M, Saji S, Suzuki A, Yamamoto Y, and Tominaga T. 1997. MDM2 in Breast Cancer. Breast Cancer 4:264-268.

Tolley DA, Parmar MK, Grigor KM, Lallemand G, Benyon LL, Fellows J, Freedman LS, Hall RR, Hargreave TB, Munson K, Newling DW, Richards B, Robinson MR, Rose MB, Smith PH, Williams JL, and Whelan P. 1996. The effect of intravesical mitomycin $\mathrm{C}$ on recurrence of newly diagnosed superficial bladder cancer: a further report with 7 years of follow up. J Urol 155:1233-1238.

van der Heijden AG, Jansen CF, Verhaegh G, O'Donnell M A, Schalken JA, and Witjes JA. 2004. The effect of hyperthermia on mitomycin-C induced cytotoxicity in four human bladder cancer cell lines. Eur Urol 46:670-674. 10.1016/j.eururo.2004.06.009

Vanden Heuvel JP, Maddox E, Maalouf SW, Iorns E, Tsui R, Denis A, Perfito N, and Errington TM. 2018. Replication Study: Systematic identification of genomic markers of drug sensitivity in cancer cells. Elife 7. 10.7554/eLife.29747

Villares GJ, Zigler M, Blehm K, Bogdan C, McConkey D, Colin D, and Bar-Eli M. 2007. Targeting EGFR in bladder cancer. World J Urol 25:573-579. 10.1007/s00345-007-0202-7

von der Maase H, Hansen SW, Roberts JT, Dogliotti L, Oliver T, Moore MJ, Bodrogi I, Albers P, Knuth A, Lippert CM, Kerbrat P, Sanchez Rovira P, Wersall P, Cleall SP, Roychowdhury DF, Tomlin I, Visseren-Grul CM, and Conte PF. 2000. Gemcitabine and cisplatin versus methotrexate, vinblastine, doxorubicin, and cisplatin in advanced or metastatic bladder cancer: results of a large, randomized, multinational, multicenter, phase III study. J Clin Oncol 18:3068-3077. 10.1200/JCO.2000.18.17.3068

Vousden KH, and Prives C. 2009. Blinded by the Light: The Growing Complexity of p53. Cell 137:413-431. 10.1016/j.cell.2009.04.037

Williamson MP, Elder PA, and Knowles MA. 1994. The spectrum of TP53 mutations in bladder carcinoma. Genes Chromosomes Cancer 9:108-118. 10.1002/gcc.2870090206

Yang W, Soares J, Greninger P, Edelman EJ, Lightfoot H, Forbes S, Bindal N, Beare D, Smith JA, Thompson IR, Ramaswamy S, Futreal PA, Haber DA, Stratton MR, Benes C, McDermott U, and Garnett MJ. 2013. Genomics of Drug Sensitivity in Cancer (GDSC): a resource for therapeutic biomarker discovery in cancer cells. Nucleic Acids Res 41:D955-961. 10.1093/nar/gks1111

Ye D, Li H, Qian S, Sun Y, Zheng J, and Ma Y. 1998. bcl-2/bax expression and p53 gene status in human bladder cancer: relationship to early recurrence with intravesical chemotherapy after resection. $J$ Urol 160:20252028; discussion 2029.

Yu C, Hequn C, Jinbo C, Feng Z, Xiongbing Z, and Jian D. 2018. Gemcitabine/cisplatin versus methotrexate/vinblastine/doxorubicin/cisplatin for muscle-invasive bladder cancer: A systematic review and meta-analysis. J Cancer Res Ther 14:1260-1265. 10.4103/0973-1482.188434 
Figure 1

Figure 1 Mutation frequency and types of TP53 in bladder cancer from the cancer Genome Atlas (TCGA) database.

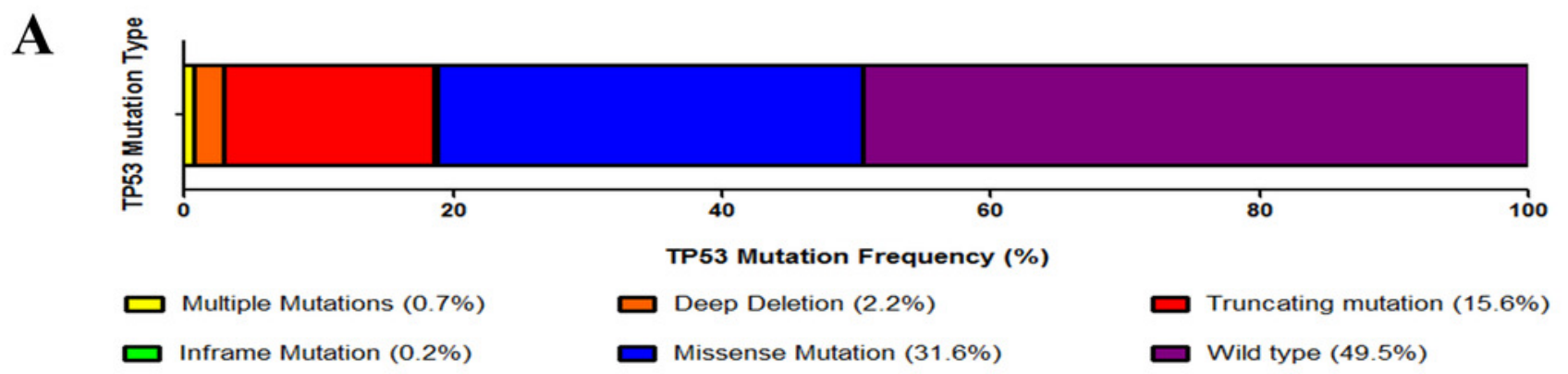

B

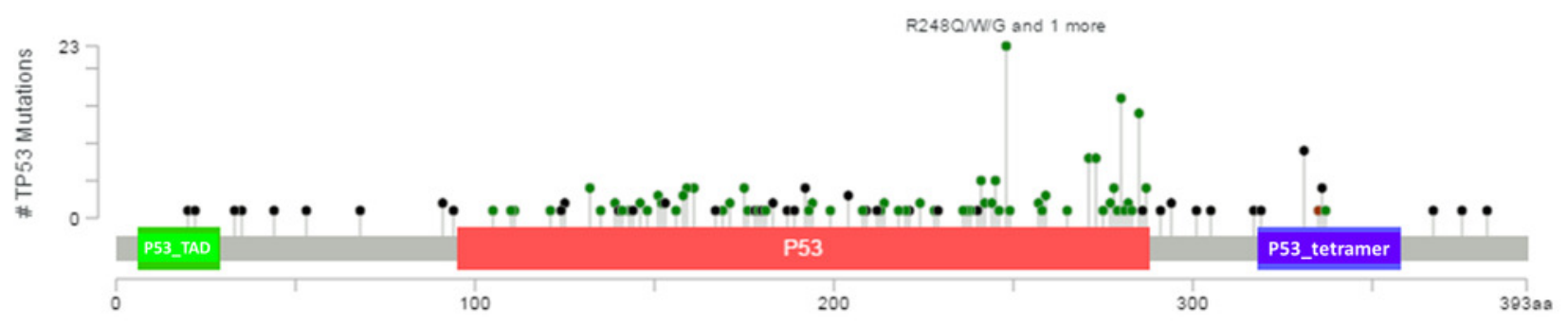

C

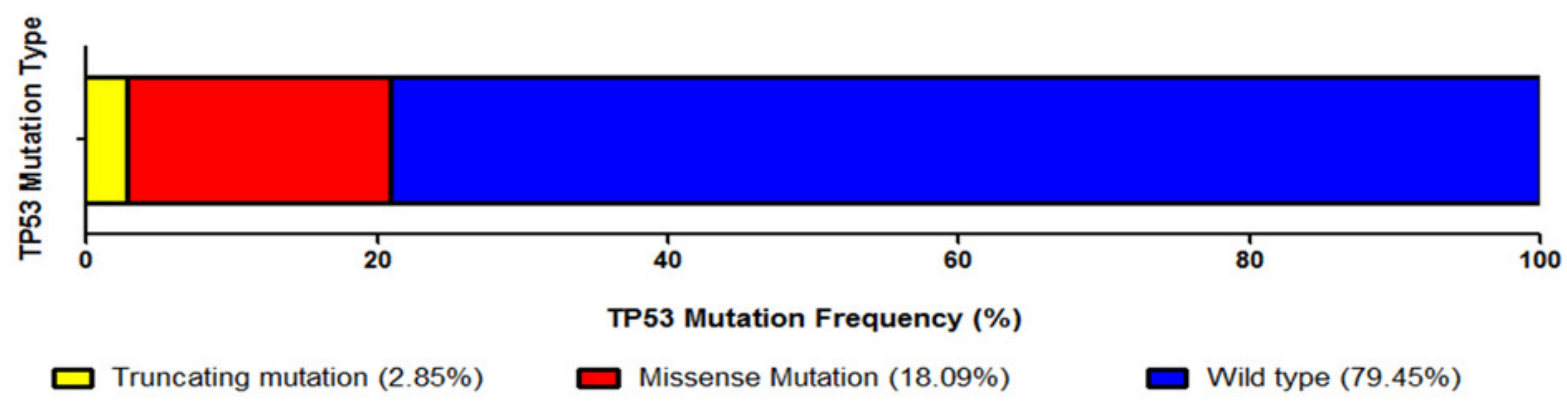

D

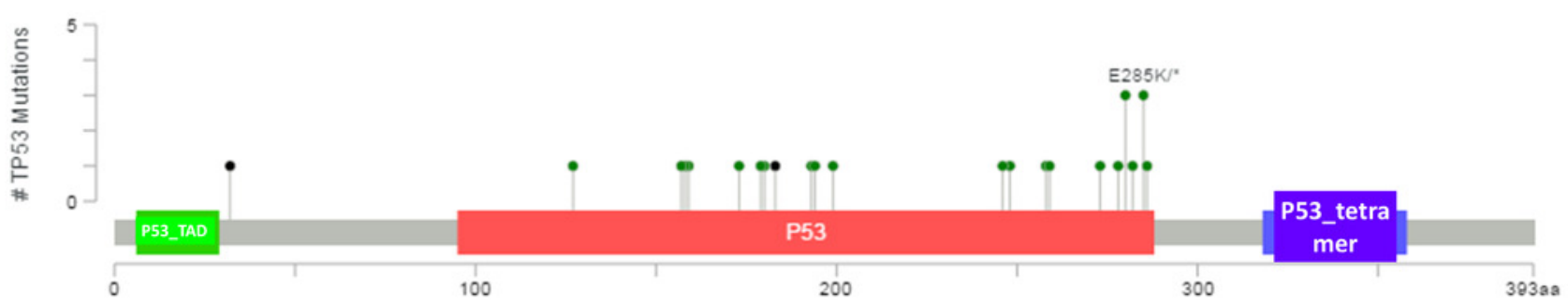


Figure 2

Figure 2 Mutation of TP53 and bladder cancer prognosis.

A

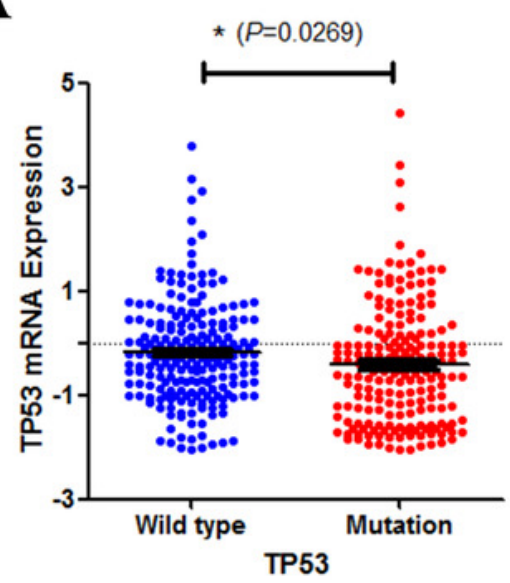

C
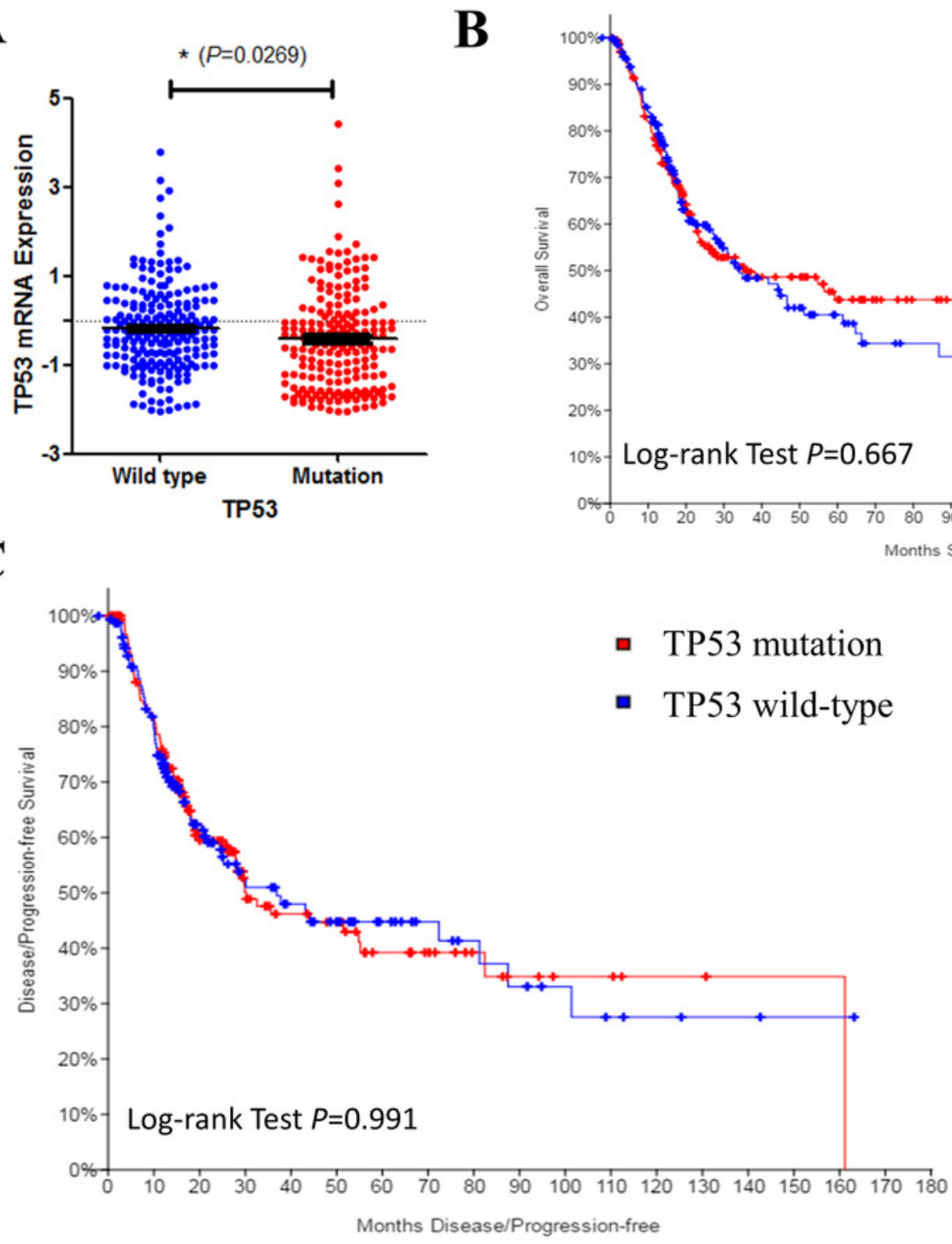

- TP53 mutation

- TP53 wild-type

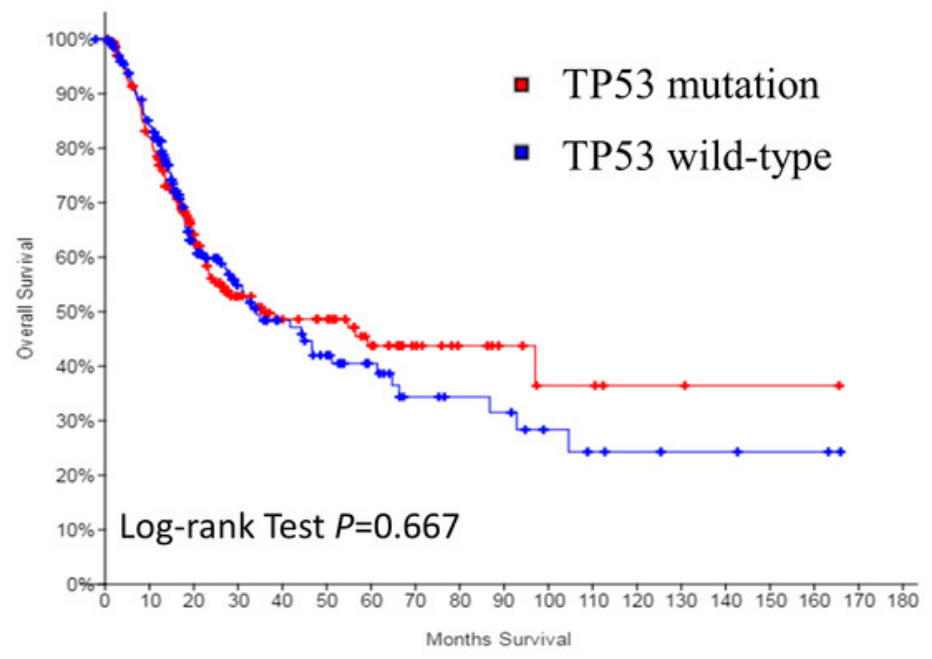


Figure 3

Figure 3 Mutation of TP53 influences drug selection of bladder cancer.

A

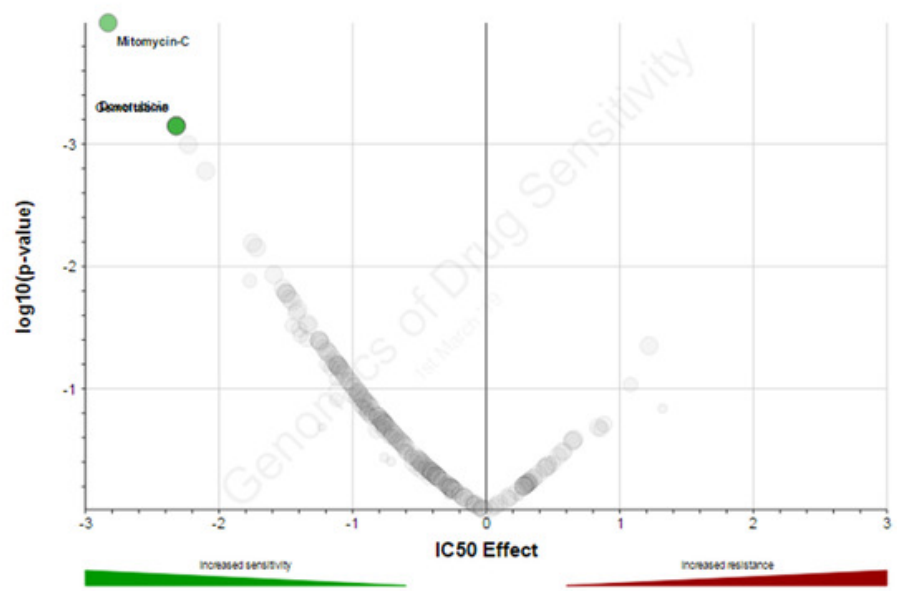

D

\section{Doxorubicin}
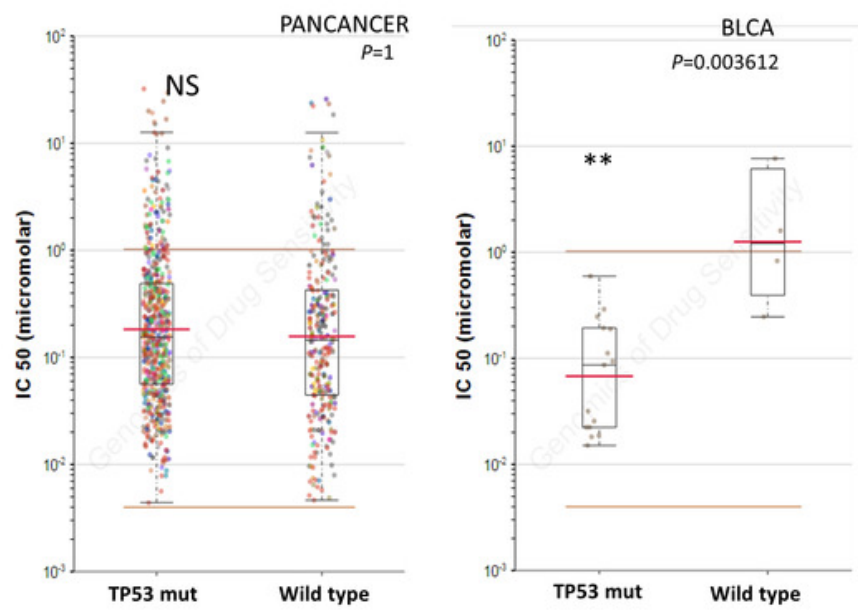

B

Mitomycin-C
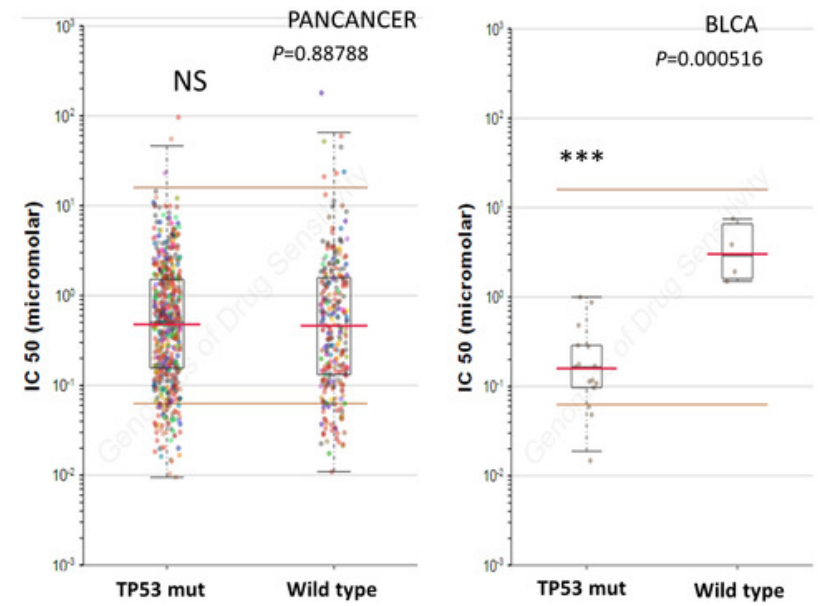

F

\section{Gemcitabine}
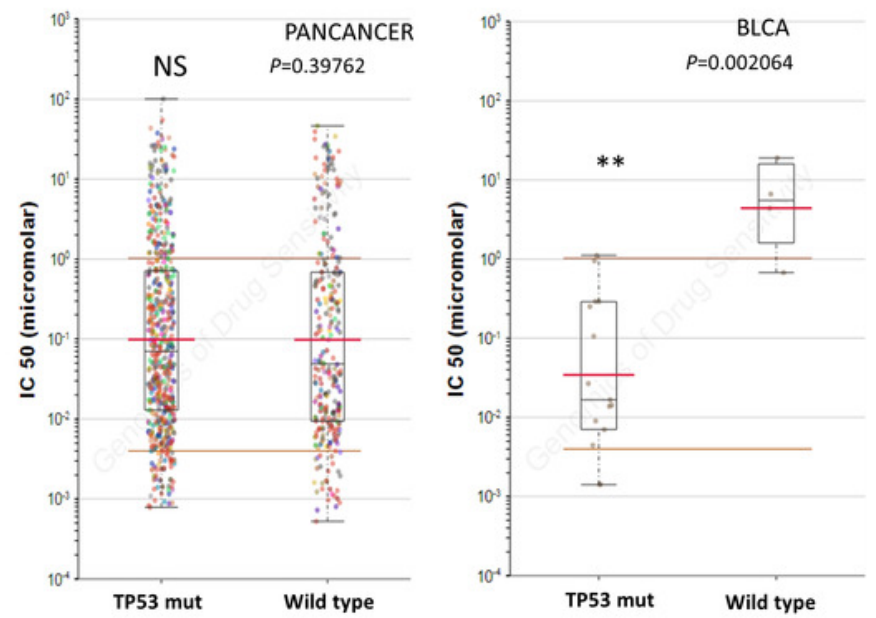
Figure 4

Figure 4 GSEA results of TP53 mutation in bladder cancer patients.

A



$\mathbf{E}$



I
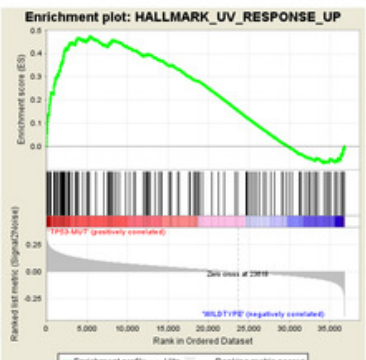

M

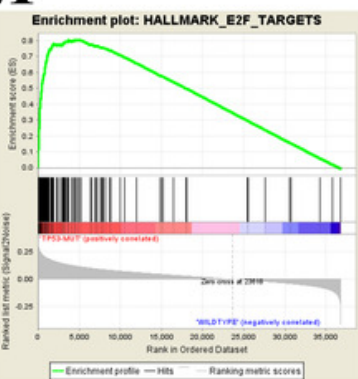

B



$\mathbf{F}$

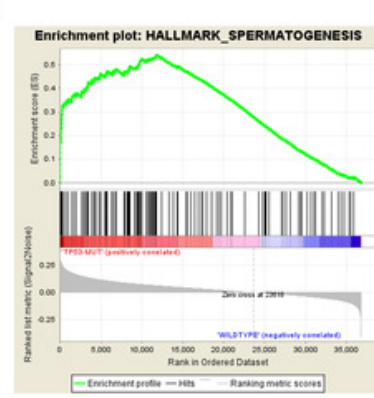

$\mathbf{J}$

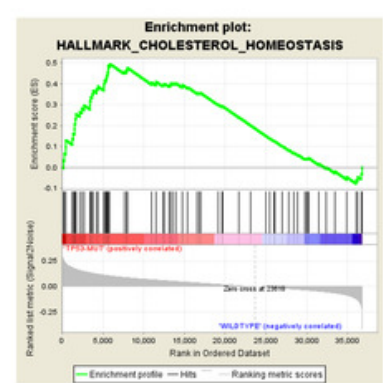

$\mathbf{N}$

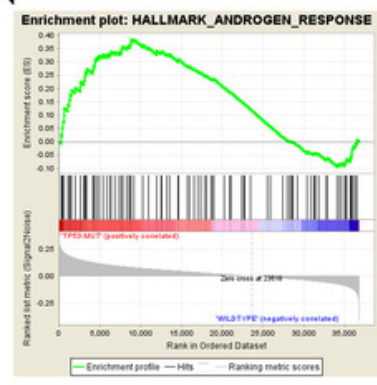

C



G

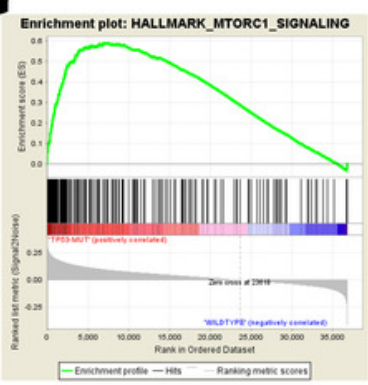

$\mathbf{K}$

Enrichment plot: HALLMARK_PROTEIN_SECRETION
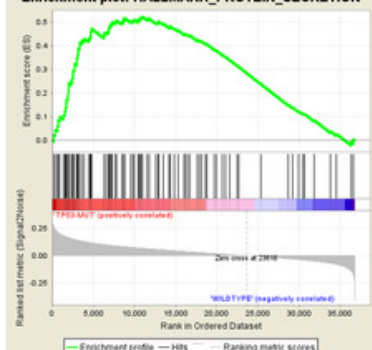

$\mathbf{O}$

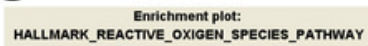

HALLMARK_REACTIVE_OXIGEN_SPECIES_PATHWAY
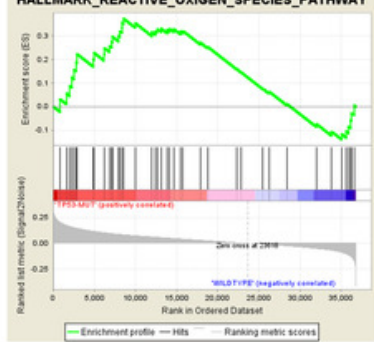

D

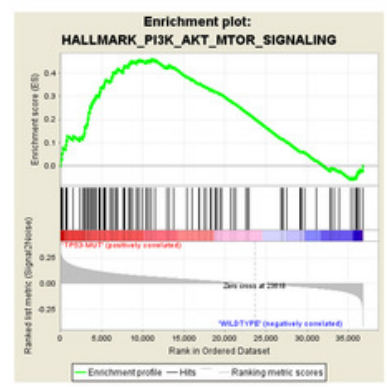

H

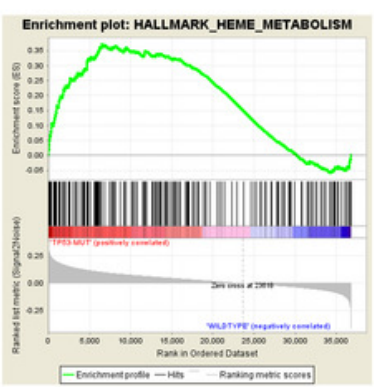

L

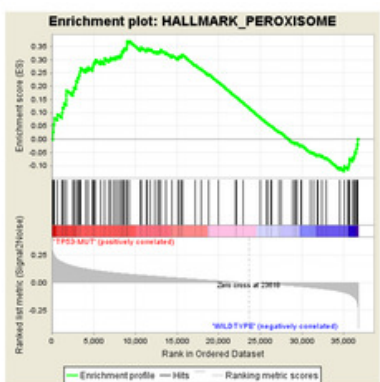

P

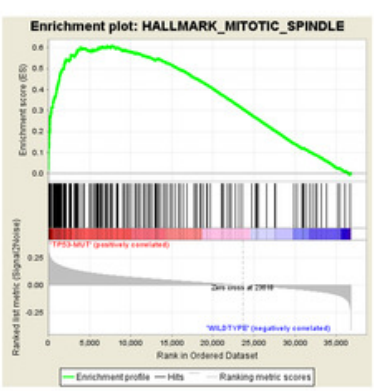


Figure 5

Figure 5 DAVID enrichment results of differentially expressed genes.

A

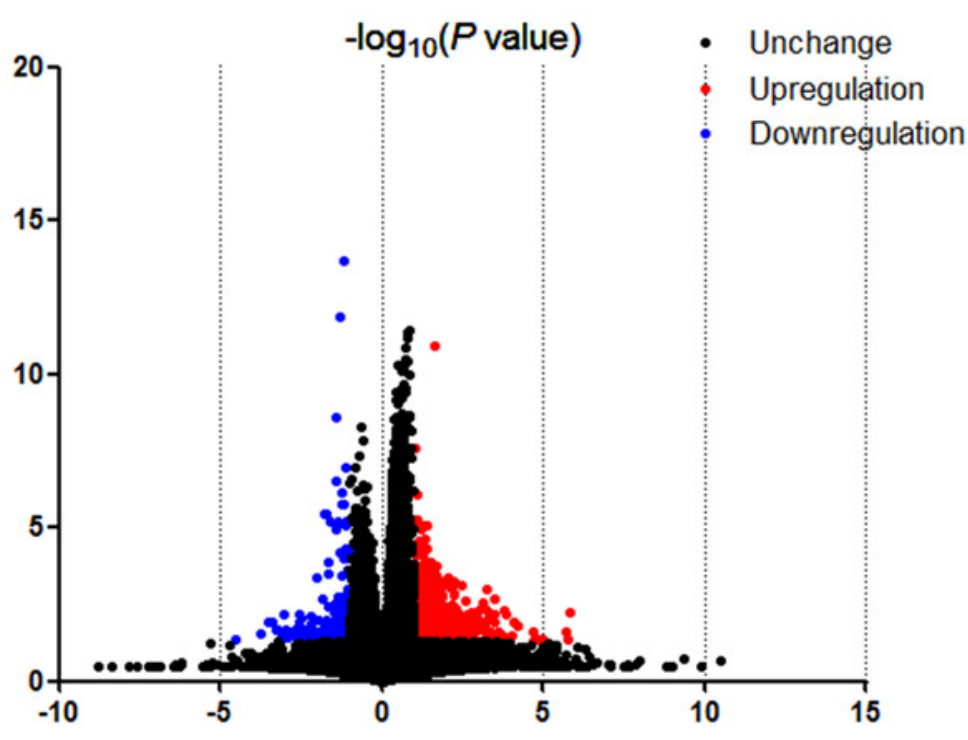

B

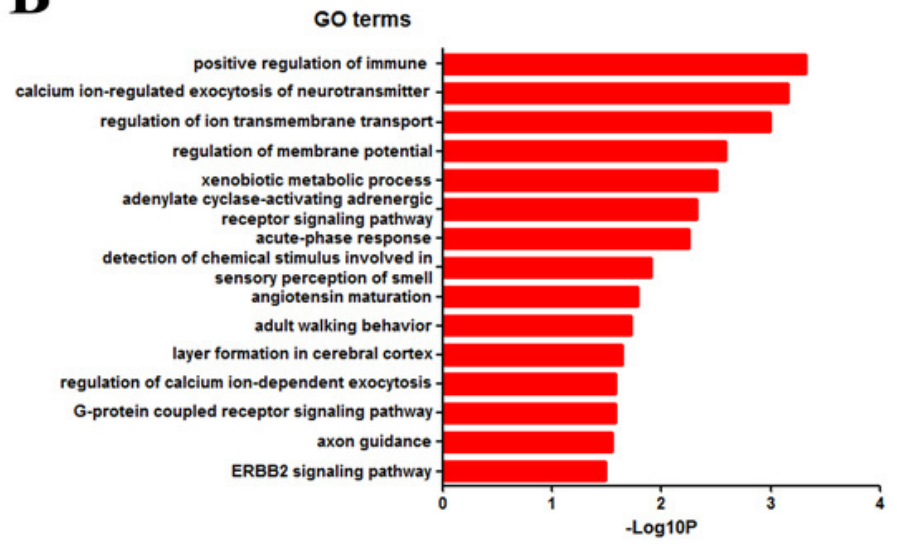

C

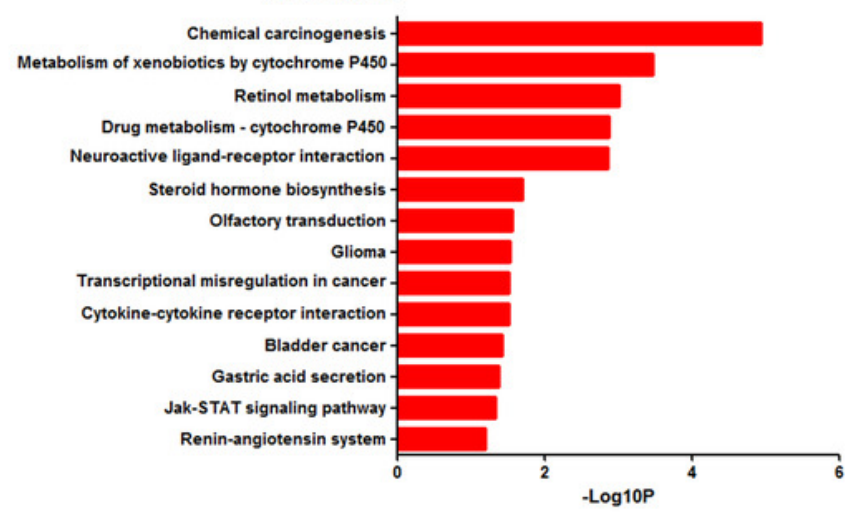




\section{Table $\mathbf{1}$ (on next page)}

Table 1. Clinical Characteristics of BLCA Patients and TP53 status in TCGA 
Table 1. Clinical Characteristics of BLCA Patients and TP53 status in TCGA

\begin{tabular}{|c|c|c|c|}
\hline \multirow{2}{*}{ Characteristics } & \multicolumn{2}{|c|}{ TP53 status } & \multirow{2}{*}{$P$ value } \\
\hline & wild type & mutated & \\
\hline Mean Age, years & 67.54 & 68.75 & \\
\hline range & $34-90$ & $44-90$ & \\
\hline Gender & & & $P=0.7853$ \\
\hline Female & 54 & 53 & \\
\hline Male & 156 & 144 & \\
\hline Tumor $\mathrm{T}$ stage & & & $P=0.6904$ \\
\hline $\mathrm{T} 1$ & 2 & 2 & \\
\hline $\mathrm{T} 2$ & 23 & 14 & \\
\hline $\mathrm{T} 2 \mathrm{a}$ & 15 & 11 & \\
\hline $\mathrm{T} 2 \mathrm{~b}$ & 30 & 26 & \\
\hline T3 & 23 & 19 & \\
\hline T3a & 30 & 41 & \\
\hline $\mathrm{T} 3 \mathrm{~b}$ & 42 & 39 & \\
\hline $\mathrm{T} 4$ & 4 & 7 & \\
\hline $\mathrm{T} 4 \mathrm{a}$ & 25 & 18 & \\
\hline $\mathrm{T} 4 \mathrm{~b}$ & 2 & 2 & \\
\hline $\mathrm{N}$ stage & & & $P=0.0166$ \\
\hline NO & 136 & 100 & \\
\hline N1 & 22 & 24 & \\
\hline $\mathrm{N} 2$ & 34 & 41 & \\
\hline N3 & 1 & 7 & \\
\hline NX & 14 & 22 & \\
\hline M stage & & & $P=0.0314$ \\
\hline M0 & 112 & 79 & \\
\hline M1 & 5 & 6 & \\
\hline MX & 92 & 110 & \\
\hline AJCC Neoplasm Disease Stage & & & $P=0.1580$ \\
\hline Stage I & 2 & 0 & \\
\hline Stage II & 69 & 60 & \\
\hline Stage III & 77 & 63 & \\
\hline Stage IV & 60 & 74 & \\
\hline Neoplasm Histologic Grade & & & $P=0.0013$ \\
\hline high & 190 & 193 & \\
\hline low & 18 & 3 & \\
\hline
\end{tabular}

